\title{
The expanding role of pertuzumab in the treatment of HER2-positive breast cancer
}

\author{
This article was published in the following Dove Press journal: \\ Breast Cancer: Targets and Therapy \\ 2I May 2015 \\ Number of times this article has been viewed
}

\section{Moya-Horno' \\ J Cortés ${ }^{1,2}$}

'Department of Medical Oncology, Instituto Oncológico Baselga, Hospital Quirón, ${ }^{2}$ Department of Medical Oncology, Vall d'Hebron Institute of Oncology, Barcelona, Spain
Correspondence: J Cortés Department of Medical Oncology, Vall d'Hebron Institute of Oncology, Passeig Vall d'Hebron I19-129, 08035 Barcelona, Spain

Tel +34 932746077

Fax +34932746059

Email jacortes@vhio.net
Abstract: Breast cancer tumors that demonstrate gene amplification or overexpression of human epidermal growth factor receptor 2 (HER2) are classified as HER2-positive. They account for approximately $15 \%$ of all breast cancers and represent an adverse prognostic factor. Over the past years, many new therapies have become available for the treatment of breast cancer. Particularly, the treatment of patients with HER2-positive breast cancer has developed with the arrival of anti-HER 2 targeted therapies that have been proven to increase survival in both the metastatic and early-stage settings of the disease. Trastuzumab, a monoclonal antibody targeting HER2, significantly improves survival in HER2-positive breast cancer. Nevertheless, it is still a challenge to evolve anti-HER2 therapies, as the disease may progress. Pertuzumab inhibits HER2 by binding to a different HER2 epitope than trastuzumab and represents a complementary mechanism of action to trastuzumab. The efficacy and safety of pertuzumab in combination with trastuzumab with or without chemotherapy have been demonstrated in both advanced and early stages of HER2-positive breast cancer. Herein, we review the available data on the use of pertuzumab for the treatment of patients with HER2positive breast cancer.

Keywords: pertuzumab, anti-HER2 targeted therapies, HER2-positive breast cancer

\section{Introduction}

Breast cancer is the most common malignancy in women worldwide. The American Cancer Society estimates that 235,000 Americans would be diagnosed with invasive breast cancer and 40,430 would have died of the disease in the US in 2014. The incidence of breast cancer has increased steadily over the past few decades, but breast cancer mortality appears to be declining, suggesting a benefit from the combination of early detection and more effective treatment. ${ }^{1,2}$ Approximately $15 \%$ of all breast cancers have gene amplification or overexpression (or both) of human epidermal growth factor receptor 2 (HER2), a tyrosine kinase transmembrane receptor, resulting in a more aggressive phenotype and adverse disease prognosis. ${ }^{3,4}$

In recent years, HER2 has become one of the most important therapeutic targets in the management of breast cancer. With the development of anti-HER 2 targeted therapies, the prognosis of HER2-positive breast cancer has improved significantly. Currently, there are four licensed anti-HER2 agents: trastuzumab, lapatinib, and more recently, pertuzumab, and trastuzumab emtansine (formerly known as T-DM1).

Trastuzumab is a recombinant humanized monoclonal antibody directed against the extracellular subdomain IV of HER2, capable of inhibiting the signaling pathway of HER2 by several mechanisms of action. The combination of systemic therapy with 
trastuzumab has significantly altered the natural history of HER2-positive breast cancer. Trastuzumab is currently the anti-HER2 treatment of choice in the first-line treatment of HER2-positive metastatic breast cancer (MBC) and in the neoadjuvant and adjuvant setting for HER2-positive early breast cancer (EBC). 5,6

Before the advent of trastuzumab, first-line treatment of HER2-positive MBC achieved a response rate (RR) of $30 \%-35 \%$, a median progression-free survival (PFS) of 5-6 months, and a median overall survival (OS) of 20-23 months. Several clinical trials have shown a significant clinical benefit with the addition of trastuzumab, showing an RR of $50 \%-72 \%$, a median PFS of 11 months-12 months, and a median OS of 25-36 months. ${ }^{5,7,8}$ Currently, taxanes (paclitaxel and docetaxel) and vinorelbine are the most common drugs used in combination with trastuzumab for the firstline treatment of HER2-positive MBC. In postmenopausal patients with HER2-positive and hormone receptor-positive $\mathrm{MBC}$, the combination of an anti-HER2 agent (trastuzumab or lapatinib) with an aromatase inhibitor may be a therapeutic alternative in those patients not candidates for chemotherapy or with low burden of tumor (paucisymptomatic and nonvisceral disease), although the effectiveness is lower than the combination of chemotherapy and trastuzumab, with an RR of $20 \%-28 \%$ and a median PFS of 8.5 months. ${ }^{9,10}$

In women with HER-2-positive tumors treated with neoadjuvant chemotherapy, the addition of neoadjuvant trastuzumab to taxanes and anthracyclines has also substantially improved the rates of pathological complete response (pCR) and disease-free survival. ${ }^{11,12}$

\section{Overview of pharmacology of pertuzumab}

Pertuzumab is a recombinant humanized monoclonal antibody directed against the extracellular dimerization domain (subdomain II) of HER2, preventing dimerization of HER2 with other members of the HER family, such as HER3, HER1, and HER4. This results in inhibited downstream signaling of two key pathways that regulate cell survival and growth (the mitogen-activated protein kinase [MAPK] pathway, and the phosphoinositide 3-kinase [PI3K] pathway), in addition to mediating antibody-dependent cell-mediated cytotoxicity. Pertuzumab is the first drug of a novel class of therapeutic antibodies known as HER dimerization inhibitors, which represent a complementary mechanism of action to trastuzumab. The addition of pertuzumab after progression to ongoing trastuzumab in xenografts synergistically increases tumor inhibition compared to trastuzumab treatment alone. ${ }^{13}$ In combination with trastuzumab and docetaxel, pertuzumab improves PFS and OS versus trastuzumab and docetaxel in the phase III CLEOPATRA trial in first-line HER2-positive MBC. ${ }^{14}$ In a substudy of CLEOPATRA evaluating potential pharmacokinetic drug-drug interaction, pertuzumab showed no impact on the pharmacokinetics of trastuzumab or docetaxel. ${ }^{15}$

Pertuzumab is presented as a concentrated solution for infusion at a concentration of $30 \mathrm{mg} / \mathrm{mL}$. One single-use vial of $14 \mathrm{~mL}$ contains $420 \mathrm{mg}$ of pertuzumab. The recommended initial dose of pertuzumab is $840 \mathrm{mg}$ administered as a 60-minute intravenous (iv) infusion, followed by a dose of $420 \mathrm{mg}$ every 3 weeks administered as an iv infusion over $30-60$ minutes. $^{16}$

\section{Efficacy, safety, and tolerability studies}

The efficacy and safety of pertuzumab in combination with trastuzumab in MBC have been demonstrated in two phase II clinical trials (the BO17929 and TOC3487 trials) and a phase III clinical trial (the CLEOPATRA trial). Two studies have evaluated this double blockade in combination with or without chemotherapy in EBC (the NeoSphere and TRYPHAENA trials) (Table 1).

The CLEOPATRA trial led to the initial approval by the US Food and Drug Administration (FDA) for pertuzumab on June 8, 2012, for use in combination with trastuzumab and docetaxel for the treatment of patients with HER2-positive MBC who have not received prior anti-HER2 therapy or chemotherapy for metastatic disease, based on significant and clinically meaningful improvements in PFS and OS, as well as an acceptable safety profile. As of August 1, 2013, pertuzumab received regulatory approval for the $\mathrm{MBC}$ indication in 53 countries.

Since the combination of pertuzumab and trastuzumab showed significant OS benefit in the metastatic setting, the FDA recently granted accelerated approval for pertuzumab in combination with trastuzumab and docetaxel as neoadjuvant treatment for patients with HER2-positive tumors greater than $2 \mathrm{~cm}$ in diameter or with positive lymph nodes. The accelerated approval was based on demonstration of an improvement in pCR in patients receiving pertuzumab, trastuzumab, and docetaxel in both the NeoSphere and TRYPHAENA trials, but no data are available demonstrating improvement in PFS or OS.

The APHINITY trial, a randomized, blinded, phase III study comparing pertuzumab, trastuzumab, and chemotherapy to trastuzumab and chemotherapy in the adjuvant treatment of patients with HER2-positive EBC, the data from 
Table I Main clinical trials evaluating pertuzumab in the treatment of HER2-positive breast cancer

\begin{tabular}{|c|c|c|c|c|c|}
\hline Trial ${ }^{\text {reference }}$ & Phase & Setting & Schema & Primary end point & Reported results \\
\hline $\begin{array}{l}\mathrm{BO} \mid 7929^{17} \\
(\mathrm{~N}=66)\end{array}$ & II & Metastatic & $\mathrm{P}+\mathrm{T}$ & $\mathrm{RR}$ & $\begin{array}{l}\text { RR: } 24.2 \% \\
\text { Other: PFS: } 5.5 \text { months; safe }\end{array}$ \\
\hline $\begin{array}{l}\text { TOC3487 } \\
(\mathrm{N}=37)\end{array}$ & II & Metastatic & $\mathrm{P}+\mathrm{T}$ & RR and safety & $\begin{array}{l}\text { RR: } 18 \% \text { in eleven patients evaluated; } \\
\text { possible increase of cardiotoxicity }\end{array}$ \\
\hline $\begin{array}{l}\text { CLEOPATRA }^{14} \\
(\mathrm{~N}=808)\end{array}$ & III & Metastatic & $\begin{array}{l}\text { Placebo+T+Doc } \\
\text { P+T+Doc }\end{array}$ & PFS & $\begin{array}{l}\text { PFS improvement in the } \mathrm{P}+\mathrm{T}+\mathrm{D} \text { oc group } \\
\text { (HR: } 0.62 ; \mathrm{P}<0.00 \mathrm{I}) \\
\text { Other: strong trend of OS in favor of the } \\
\mathrm{P}+\mathrm{T}+\text { Doc group; similar safety profile }\end{array}$ \\
\hline $\begin{array}{l}\text { NeoSphere }{ }^{28} \\
(\mathrm{~N}=4 \mid 7)\end{array}$ & II & Neoadjuvant & $\begin{array}{l}\text { T+Doc } \\
\text { P+T+Doc } \\
\text { P+T } \\
\text { P+Doc }\end{array}$ & $\mathrm{PCR}$ & $\begin{array}{l}\mathrm{PCR} \text { improvement in the } \mathrm{P}+\mathrm{T}+\mathrm{Doc} \\
\text { group ( } 45.8 \% \text { ) } \\
\text { Other: similar safety profile }\end{array}$ \\
\hline $\begin{array}{l}\text { TRYPHAENA }^{29} \\
(\mathrm{~N}=225)\end{array}$ & II & Neoadjuvant & $\begin{array}{l}\mathrm{P}+\mathrm{T}+\mathrm{FEC} \rightarrow \mathrm{P}+\mathrm{T}+\mathrm{Doc} \\
\mathrm{FEC} \rightarrow \mathrm{P}+\mathrm{T}+\mathrm{Doc} \\
\mathrm{P}+\mathrm{T}+\text { Doc }+\mathrm{CP}_{\mathrm{P}}\end{array}$ & Cardiac safety & $\begin{array}{l}\text { Low incidence of symptomatic LVSD and } \\
\text { significant declines in LVEF } \\
\text { Other: similar pCR rates across groups } \\
(57.3 \%-66.2 \%)\end{array}$ \\
\hline
\end{tabular}

Abbreviations: P, pertuzumab; T, trastuzumab; Doc, docetaxel; FEC, fluorouracil/epirubicin/cyclophosphamide; Cp, carboplatin; RR, response rate; PFS, progression-free survival; PCR, pathological complete response; HR, hazard ratio; OS, overall survival; LVSD, left ventricular systolic dysfunction; LVEF, left ventricular ejection fraction.

which are expected in 2016, might provide a robust body of data for the combination of pertuzumab, trastuzumab, and chemotherapy in the early-stage setting.

\section{Efficacy of pertuzumab in MBC}

The phase II BO17929 and TOC3487 trials were conducted in patients with HER-2-positive MBC, which had received at least three lines of prior chemotherapy and had experienced progression with trastuzumab, with the objective of evaluating the efficacy and safety of the combination of pertuzumab and trastuzumab without chemotherapy. In the BO17929 trial, the combination of pertuzumab and trastuzumab showed a significant antitumor activity in 66 patients, with an RR of $24.2 \%$ and a median PFS of 5.5 months. ${ }^{17}$ To define whether this clinical benefit was a result of the combination of pertuzumab and trastuzumab or pertuzumab alone, a cohort of 29 patients whose disease was initially treated with pertuzumab in monotherapy, with the addition of trastuzumab at the time of tumor progression, was recruited. The results of the cohort of 29 patients treated with pertuzumab in monotherapy were poor, with an RR of $3.4 \%$, but with the addition of trastuzumab, the RR was $17.6 \% .{ }^{18}$ The TOC 3487 trial was planned to include 37 patients but was stopped early because of a possible increase of cardiotoxicity with the dual HER 2 blockade. The RR was $18 \%$ in the eleven patients evaluated. ${ }^{19}$ The main data on efficacy of pertuzumab in first-line treatment of HER2-positive MBC were obtained from the CLEOPATRA trial. ${ }^{14}$ This is a randomized, double-blind, phase III study comparing placebo, trastuzumab, and docetaxel versus pertuzumab, trastuzumab, and docetaxel for patients with HER2- positive MBC with no previous history of chemotherapy or biological treatment for their disease. Patients could have received one hormonal treatment for MBC before randomization. They could also have received neoadjuvant or adjuvant chemotherapy with or without trastuzumab, provided, in this case, that disease-free interval was at least 12 months from completion of the neoadjuvant or adjuvant treatment to diagnosis of MBC. Eligible patients were aged 18 years or older, had measurable or nonmeasurable disease, a left ventricular ejection fraction (LVEF) $\geq 50 \%$ at baseline, and an Eastern Cooperative Oncology Group (ECOG) performance status of 0 or 1 . Exclusion criteria were central nervous system (CNS) metastases and previous exposure to doxorubicin exceeding $360 \mathrm{mg} / \mathrm{m}^{2}$. Patients received placebo/pertuzumab ( $840 \mathrm{mg}$ iv loading dose, then $420 \mathrm{mg}$ iv every 3 weeks), trastuzumab ( $8 \mathrm{mg} / \mathrm{kg}$ iv loading dose, then $6 \mathrm{mg} / \mathrm{kg}$ iv every 3 weeks), and docetaxel ( $75 \mathrm{mg} / \mathrm{m}^{2}$ iv every 3 weeks, $100 \mathrm{mg} /$ $\mathrm{m}^{2}$ iv every 3 weeks if tolerated). Placebo/pertuzumab and trastuzumab were administered until disease progression or unmanageable toxic effects. Dose reductions of pertuzumab and trastuzumab were not allowed. The dose of docetaxel could be reduced by $25 \%$ to manage toxicity. At least six cycles of docetaxel were recommended. Fewer cycles of the drug were permitted if disease progression or unmanageable toxicity occurred. The primary end point of the study was independent assessment of PFS. The secondary end points were investigator-assessed PFS, OS, objective RR, and safety. Of the 808 patients enrolled in the trial, 406 were randomly assigned to receive placebo, trastuzumab, and docetaxel (control group) and 402 received pertuzumab, trastuzumab, and 
docetaxel (pertuzumab group). The addition of pertuzumab to trastuzumab and docetaxel provided a statistically significant improvement in PFS compared to placebo, trastuzumab, and docetaxel treatment. The median independently assessed PFS was increased by 6.1 months, from 12.4 months in the control group to 18.5 months in the pertuzumab group (hazard ratio [HR]: 0.62; 95\% confidence interval [CI]: 0.51-0.75; $P<0.001)$. Among the 88 patients who had received neoadjuvant or adjuvant chemotherapy with trastuzumab, the median independently assessed PFS was 10.4 months in the control group and 16.9 months in the pertuzumab group (HR: 0.62; 95\% CI: 0.35-1.07). Among the 288 patients who had received neoadjuvant or adjuvant chemotherapy without trastuzumab, the median independently assessed PFS was 12.6 months in the control group and 21.6 months in the pertuzumab group (HR: $0.60 ; 95 \% \mathrm{CI}$ : $0.43-0.83$ ). Investigator-assessed median PFS was 12.4 months in the control group and 18.5 months in the pertuzumab group (HR: 0.65; 95\% CI: 0.54-0.78; $P<0.001$ ). The improvement in PFS was independent of biomarker subgroups (HR: $<1.0$ ), including estrogen receptor-negative and -positive subgroups. High levels of HER2 and HER3 mRNA and HER2 protein, wild-type PIK3CA, and low serum HER2 extracellular domain, showed a significantly better prognosis $(P<0.05)$. PIK3CA represented the greatest prognostic factor, with longer PFS in patients with wild-type versus mutated PIK3CA tumors in both the control (13.8 months versus 8.8 months) and pertuzumab (21.8 months versus 12.5 months) groups. ${ }^{20}$ At the time of the independent assessment of PFS, the interim analysis of OS showed a trend in favor of the pertuzumab group, but this was not significant. After a follow-up of 30 months, the results showed a statistically significant improvement in OS favoring the pertuzumab-containing arm, with a $34 \%$ reduction in the risk of death (HR: $0.66 ; 95 \% \mathrm{CI}$ : $0.52-0.84 ; P=0.0008) .{ }^{21}$ At median follow-up of 50 months, the statistically significant improvement in OS in favor of the pertuzumab group was maintained (HR: $0.68 ; 95 \% \mathrm{CI}$ : $0.56-0.84 ; P=0.0002) .{ }^{22}$ The median OS was 40.8 months in the control group and 56.5 months in the pertuzumab group, with difference of 15.7 months. The objective RR in the CLEOPATRA trial was $69.3 \%$ in the control group and $80.2 \%$ in the pertuzumab group. The difference in RR was 10.8 percentage points ( $95 \% \mathrm{CI}: 4.2-17.5 ; P=0.001)$. As OS at the interim analysis did not cross the stopping boundary for significance, the statistical test result for objective RR was considered to be exploratory. An analysis of the incidence and time to development of CNS metastases in patients from the CLEOPATRA trial showed that the proportion of patients developing CNS as first site of disease progression was similar between the control group ( 51 of 406, 12.6\%) and the pertuzumab group ( 55 of $402,13.7 \%$ ). The median time to progression in the CNS was 11.9 months in the control group and 15.0 months in the pertuzumab group (HR: 0.58; 95\% CI: $0.39-0.85 ; P=0.0049)$. Median OS in patients who developed CNS metastases showed a trend in favor of the pertuzumab group, being 26.3 months versus 34.4 months in the control and pertuzumab groups, respectively (HR: 0.66; 95\% CI: 0.39-1.11). The difference observed was not statistically significant for the log-rank test $(P=0.1139)$ but was significant for the Wilcoxon test $(P=0.0449){ }^{23}$

In the setting of $\mathrm{MBC}$, phase II trials have also found activity and tolerability for other regimens combining pertuzumab and trastuzumab together with other active cytotoxics (paclitaxel and vinorelbine). ${ }^{24,25}$ The interim analysis of the single-arm phase IIIb trial PERUSE evaluating safety and efficacy of first-line pertuzumab in combination with trastuzumab and a broader range of taxanes (investigator's choice of docetaxel, paclitaxel, or nab-paclitaxel) in patients with HER2-positive locally recurrent cancer or MBC is also consistent with previous clinical experience of the combination of pertuzumab, trastuzumab, and a taxane. ${ }^{26}$ The combination of pertuzumab and T-DM1 in the treatment of patients with HER2-positive MBC has been reported in a phase II trial, showing RR of up to $57 \%$ with no unexpected toxicities. ${ }^{27}$ Further clinical trials of pertuzumab plus other anti-HER2 therapies beyond trastuzumab in the treatment of patients with HER2-positive MBC are warranted.

As commented before, the use of trastuzumab and pertuzumab in trastuzumab-resistant patients has been explored in phase II studies. ${ }^{17,18,24}$ Based on the promising activity of this combination, a randomized phase III trial exploring the use of capecitabine and trastuzumab with or without pertuzumab (the PHEREXA trial) will define the role of pertuzumab in trastuzumab-resistant patients.

In view of these upcoming data, for patients progressing after treatment with trastuzumab-based therapy without pertuzumab, a line of therapy containing both trastuzumab and pertuzumab, with or without a cytotoxic agent (such as vinorelbine or taxane), might be considered.

Further research is needed to determine the ideal strategy for anti-HER2 therapy sequence.

\section{Efficacy of pertuzumab in EBC}

The approval by the FDA for pertuzumab in the neoadjuvant setting is based on the results of the NeoSphere and the TRYPHAENA phase II trials. ${ }^{28,29}$ 
In the NeoSphere trial, 417 patients with HER2-positive, operable (T2-3, N0-1, M0), locally advanced (T2-3, N2-3, M0; T4a-c, any N, M0), or inflammatory (T4d, any N, M0) breast cancer with primary tumors larger than $2 \mathrm{~cm}$ in diameter were randomized in a 1:1:1:1 ratio to receive trastuzumab plus docetaxel, or pertuzumab and trastuzumab plus docetaxel, or pertuzumab and trastuzumab, or pertuzumab plus docetaxel. Of the patients who received pertuzumab plus trastuzumab and docetaxel, $45.8 \%$ (95\% CI: 36.1-55.7) achieved pCR, compared with only $29 \%$ (95\% CI: 20.6-38.5) of patients who achieved pCR on the trastuzumab plus docetaxel regimen $(P=0.0063)$.

The TRYPHAENA was a phase II, randomized, multicenter trial designed to evaluate the safety and tolerability of trastuzumab and pertuzumab in combination with either anthracycline-based or carboplatin-based neoadjuvant chemotherapy. A total of 225 patients with HER2-positive, locally advanced (T2-3, N2-3, M0; T4a-c, any N, M0), inflammatory (T4d, any N, M0), or early-stage breast cancer (tumors greater than $2 \mathrm{~cm}$ in diameter) were enrolled and randomized in a 1:1:1 ratio to receive six cycles of neoadjuvant therapy with FEC (fluorouracil/epirubicin/cyclophosphamide) plus trastuzumab and pertuzumab followed by docetaxel, trastuzumab, and pertuzumab; or FEC followed by docetaxel, trastuzumab, and pertuzumab; or docetaxel, carboplatin, and trastuzumab along with pertuzumab. Based on the assessment of $\mathrm{pCR}$, all three regimens seemed active. The reported $\mathrm{pCR}$ ranged from $57.3 \%$ to $66.2 \%$. The highest pCR $(66.2 \%)$ was observed in patients who received pertuzumab, trastuzumab, docetaxel, and carboplatin chemotherapy. Correlation of biomarker subgroups with the achievement of a pCR was analyzed in the TRYPHAENA study. Levels of HER $2 \mathrm{mRNA}$ and protein were associated with the $\mathrm{pCR}$ rates when data from all groups were pooled. These findings, consistent with results from the CLEOPATRA trial, highlight HER2 as the most clinically relevant biomarker for selecting patients for HER2-targeted therapies in the absence of signals for other markers analyzed. ${ }^{30}$

\section{Safety and tolerability of pertuzumab}

The initial safety data of pertuzumab were obtained from the phase II trials BO17929 and TOC3487 evaluating the combination of pertuzumab and trastuzumab without chemotherapy. In the BO17929 trial, the combination of pertuzumab and trastuzumab was well tolerated, and adverse events were mild or moderate, the most frequent being diarrhea (64\%), asthenia (33\%), and nausea (27\%). ${ }^{17}$ In the TOC3487 trial, six $(54 \%)$ patients experienced a reduction in LVEF. Three patients had grade 1, two had grade 2, and one had grade 3 left ventricular systolic dysfunction (LVSD). All patients had previous exposure to anthracyclines, the majority had exposure to adjuvant trastuzumab, and all of them had had trastuzumab for treatment of metastatic disease. Because cardiac safety was a major concern, accrual of this study was stopped before the planned enrollment of 37 patients. ${ }^{19}$

The main safety data of pertuzumab were evaluated in patients who received at least one dose of the drug in the CLEOPATRA trial. ${ }^{14}$ The addition of pertuzumab to trastuzumab and docetaxel was well tolerated, and the adverse-event profile was generally balanced between the control group and the pertuzumab group. The most common adverse reactions reported in the pertuzumab group, compared to the control group, were diarrhea (66.8\% versus $46.3 \%)$, rash $(33.7 \%$ versus $24.2 \%$ ), mucosal inflammation (27.8\% versus $19.9 \%$, febrile neutropenia (13.8\% versus $7.6 \%)$, and dry skin $(10.6 \%$ versus $4.3 \%$ ). However, peripheral edema and constipation were greater in the control group (23.1\% versus $30 \%$, and $15 \%$ versus $24.9 \%$, respectively). The incidence of cardiac adverse events was slightly higher in the control group (16.4\%) compared with the pertuzumab group (14.5\%), LVSD (all grades) being the most frequently reported event $(8.3 \%$ versus $4.4 \%$ in the control and pertuzumab groups, respectively). Symptomatic LVSD occurred in a low proportion of patients $(1.8 \%$ versus $1.0 \%$ in the control and pertuzumab groups, respectively) and had resolved at data cutoff in the majority of cases. Declines in LVEF by $\geq 10 \%$ points from baseline and up to $<50 \%$ were reported in $6.6 \%$ and $3.8 \%$ of patients in the control and pertuzumab groups, respectively. Of these patients, $72 \%$ (control group) and $86.7 \%$ (pertuzumab group) recovered to a value $\geq 50 \% .{ }^{31}$ Health-related quality of life, measured as the time to $\mathrm{a} \geq 5$-point decline in the Trial Outcome Index-Physical/Functional/Breast (TOI-PFB) of the Functional Assessment of Cancer Therapy-Breast (FACT-B) questionnaire, was not different between the two treatment groups of the CLEOPATRA trial. The median times to TOIPFB deterioration were 18.4 weeks and 18.3 weeks in the control and pertuzumab groups, respectively, representing approximately six cycles (HR: 0.97 ; 95\% CI: 0.81-1.16; $P=0.7161)$. The addition of pertuzumab to trastuzumab and docetaxel also prolonged the time to worsening of breast cancer symptoms. The analysis yielded a median time to deterioration in breast cancer symptoms of 18.3 weeks in the control group compared with 26.7 weeks in the pertuzumab group (HR: $0.77 ; 95 \%$ CI: $0.64-0.93 ; P=0.0061){ }^{32}$

In the NeoSphere trial, evaluating the efficacy and safety of neoadjuvant pertuzumab in combination with trastuzumab 
and docetaxel in women with HER2-positive EBC, no substantial differences in tolerability between the treatment groups were seen. ${ }^{28}$ In the TRYPHAENA trial, whose main objective was to evaluate the tolerability, particularly with respect to cardiac function, of three neoadjuvant treatment regimens combining pertuzumab with trastuzumab and either standard anthracycline- or platinum-based chemotherapy for the treatment of HER2-positive EBC, the combination of trastuzumab and pertuzumab was generally well tolerated regardless of whether it was given sequentially or concomitantly with anthracycline-based, or combined with carboplatin-based, chemotherapy. Pertuzumab did not increase the rate of cardiac dysfunction observed in combinations of trastuzumab plus standard chemotherapy. ${ }^{29}$

A meta-analysis of six randomized trials evaluating the cardiac toxicity between anti-HER2 monotherapy (trastuzumab, or pertuzumab, or lapatinib) and anti-HER2 combination therapy (trastuzumab and pertuzumab, or trastuzumab and lapatinib) with or without chemotherapy in breast cancer, regardless of treatment setting (metastatic or neoadjuvant), showed that dual HER2 blockade does not significantly increase the risk of cardiac adverse events compared with anti-HER2 monotherapy. ${ }^{33}$ Congestive heart failure grade $\geq 3$ occurred in $0.88 \%(95 \%$ CI: 0.47-1.64) of patients treated with combined anti-HER2 therapy, compared with $1.49 \%$ (95\% CI: 0.98-2.23) of patients in the monotherapy group. The incidence of decline in LVEF to $<50 \%$ was $3.1 \%$ (95\% CI: $2.2-4.4)$ and $2.9 \%$ (95\% CI: 2.1-4.1), respectively. The odds ratios of congestive heart failure and LVEF decline between anti-HER2 combination and monotherapy were 0.58 (95\% CI: $0.26-1.27 ; P=0.17)$ and 0.88 (95\% CI: $0.53-1.48 ; P=0.64)$, respectively.

\section{Discussion}

The addition of pertuzumab to the combination of trastuzumab and docetaxel for the first-line treatment of patients with HER2-positive MBC significantly improves PFS, OS, and RR, with no increase in cardiac toxic effects, as demonstrated in the CLEOPATRIA trial. The benefit of pertuzumab-trastuzumabdocetaxel therapy with respect to PFS is observed across all subgroups, except for patients with nonvisceral metastatic disease, for which no statistically significant difference in PFS is observed. In this subgroup of patients, the wide CI (HR: 0.96; 95\% CI: 0.61-1.52) indicates an imprecise estimate that is associated with fewer events, which may be caused by the small sample size and the different biological behaviors of this population. Therefore, it would not be advisable with the available data to restrict the indication of pertuzumab to the subgroup of patients with visceral disease.
The majority of patients included in the CLEOPATRA trial were trastuzumab naive, and if previously treated with trastuzumab ( $10 \%$ of patients), a 12-month disease-free interval was required. Therefore, the trial cannot support the use of this combination in patients progressing to trastuzumabbased neoadjuvant or adjuvant treatment within the first 12 months of completion of trastuzumab. The exploratory analysis of the subgroup of patients who had previously received neoadjuvant or adjuvant chemotherapy with trastuzumab indicates that the efficacy results are similar to those of the overall population.

Although patients in the CLEOPATRA trial were all treated with docetaxel, the use of paclitaxel or nab-paclitaxel with pertuzumab and trastuzumab is also reasonable, particularly for patients who might not be good candidates for docetaxel. ${ }^{24,26}$ Although it is likely that other chemotherapy agents can be combined safely and effectively with trastuzumab and pertuzumab, ${ }^{25}$ the use of alternative regimens would be supported only by limited data and should generally be avoided until additional data are available.

At the present time, there is insufficient evidence to make a strong recommendation for the use of pertuzumab beyond first-line treatment of HER2-positive MBC, and T-DM1 should be recommended as a second-line treatment for patients with HER2-positive MBC progressing during or after first-line HER2-targeted therapy. ${ }^{34,35}$

The clinical trials NeoSphere and TRYPHAENA studying the neoadjuvant treatment of HER2-positive EBC provide evidence for the safety and efficacy of the combination of pertuzumab plus trastuzumab plus either a standard anthracycline-based, or platinum-based, chemotherapy. The approval of pertuzumab in the neoadjuvant setting is based on demonstration of an improvement in pCR, despite lack of OS data. A pooled analysis of clinical trials of neoadjuvant treatment for breast cancer showed that patients who attain pCR defined as ypT0, ypN0 or ypT0/is, and ypN0 have improved OS (ypT0, ypN0: HR 0.36, 95\% CI 0.30-0.44; ypT0/is, ypN0: HR 0.36, 95\% CI $0.31-0.42) .{ }^{36}$ The association between pCR and OS was strongest in patients with triple-negative and HER2positive tumors.

Available data on the tolerability of dual anti-HER2 therapy, in both metastatic and neoadjuvant settings, provide reassuring information that combining anti-HER2 therapies is safe and does not increase cardiac toxicity compared to single-agent anti-HER2 therapy. Accurate selection of patients and cardiac monitoring is essential to manage potential cardiotoxicity. 


\section{Conclusion}

Pertuzumab is a HER2-targeted monoclonal antibody that provides a more complete blockade of the HER pathway in combination with trastuzumab (dual blockade), resulting in augmented anticancer activity in patients with HER2-positive breast cancer.

Based on the available published data, candidates for treatment with pertuzumab in combination with trastuzumab and docetaxel are those patients with HER2-positive MBC who have not received prior anti-HER2 therapy or chemotherapy for metastatic disease, with good performance status (ECOG 0-1) and a LVEF $\geq 50 \%$ at baseline. It seems reasonable to assume a certain degree of effectiveness of pertuzumab also in those patients with a disease-free interval between 6 months and 12 months after completion of neoadjuvant or adjuvant trastuzumab-based therapy. In patients with HER2-positive MBC who might not be good candidates for docetaxel, the use of paclitaxel or nab-paclitaxel with pertuzumab and trastuzumab is also reasonable for first-line treatment. In combination with trastuzumab and chemotherapy, pertuzumab has also shown substantial activity and good tolerability as a neoadjuvant therapy in patients with high-risk HER2-positive EBC.

Research is needed to address further indications of pertuzumab beyond first-line treatment of patients with HER2-positive MBC and as part of a complete neoadjuvant treatment regimen for patients with HER-2 positive EBC.

\section{Disclosure}

J Cortés acts as a consultant for Roche, and Celgene and has received honoraria from Roche, Celgene, Eisai, and Novartis. I Moya-Horno has no conflicts of interest to disclose.

\section{References}

1. Siegel R, Ma J, Zou Z, Jemal A. Cancer statistics, 2014. CA Cancer J Clin. 2014;64(1):9-29.

2. Berry DA, Cronin KA, Plevritis SK, et al; Cancer Intervention and Surveillance Modeling Network (CISNET) Collaborators. Effect of screening and adjuvant therapy on mortality from breast cancer. $N E n g l$ J Med. 2005;353:1784-1792.

3. Wolff AC, Hammond ME, Schwartz JN, et al; American Society of Clinical Oncology/College of American Pathologists. American Society of Clinical Oncology/College of American Pathologists guideline recommendations for human epidermal growth factor receptor 2 testing in breast cancer. Arch Pathol Lab Med. 2007;131:18-43.

4. Ross JS, Slodkowska EA, Symmans WF, Pusztai L, Ravdin PM, Hortobagyi GN. The HER-2 receptor and breast cancer: ten years of targeted anti-HER-2 therapy and personalized medicine. Oncologist. 2009; $14: 320-368$.

5. Slamon DJ, Leyland-Jones B, Shak S, et al. Use of chemotherapy plus a monoclonal antibody against HER2 for metastatic breast cancer that overexpresses HER2. N Engl J Med. 2001;344(11):783-792.

6. Slamon D, Eiermann W, Robert N, et al; Breast Cancer International Research Group. Adjuvant trastuzumab in HER2-positive breast cancer. N Engl J Med. 2011;365(14):1273-1283.
7. Marty M, Cognetti F, Maraninchi D, et al. Randomized phase II trial of the efficacy and safety of trastuzumab combined with docetaxel in patients with human epidermal growth factor receptor 2-positive metastatic breast cancer administered as first-line treatment: the M77001 study group. J Clin Oncol. 2005;23(19):4265-4274.

8. Andersson M, Lidbrink E, Bjerre K, et al. Phase III randomized study comparing docetaxel plus trastuzumab with vinorelbine plus trastuzumab as first-line therapy of metastatic or locally advanced human epidermal growth factor receptor 2-positive breast cancer: the HERNATA study. J Clin Oncol. 2011;29(3):264-271.

9. Johnston S, Pippen J, Pivot X, et al. Lapatinib combined with letrozole versus letrozole and placebo as first-line therapy for postmenopausal hormone receptor-positive metastatic breast cancer. J Clin Oncol. 2009;27(33):5538-5546.

10. Kaufman B, Mackey JR, Clemens MR, et al. Trastuzumab plus anastrozole versus anastrozole alone for the treatment of postmenopausal women with human epidermal growth factor receptor 2-positive, hormone receptor-positive metastatic breast cancer: results from the randomized phase III TAnDEM study. J Clin Oncol. 2009;27(33):5529-5537.

11. Buzdar AU, Ibrahim NK, Francis D, et al. Significantly higher pathologic complete remission rate after neoadjuvant therapy with trastuzumab, paclitaxel, and epirubicin chemotherapy: results of a randomized trial in human epidermal growth factor receptor 2-positive operable breast cancer. J Clin Oncol. 2005;23:3676-3685.

12. Gianni L, Eiermann W, Semiglazov V, et al. Neoadjuvant chemotherapy with trastuzumab followed by adjuvant trastuzumab versus neoadjuvant chemotherapy alone, in patients with HER2-positive locally advanced breast cancer (the NOAH trial): a randomized controlled superiority trial with a parallel HER2-negative cohort. Lancet. 2010;375:377-384.

13. Friess T, Thier M, Scheuer W, et al. Combination treatment with pertuzumab and trastuzumab against Calu-3 human NSCLC xenograft tumors is superior to monotherapy. Presented at: The 17th Annual Meeting of the American Association for Cancer Research-National Cancer Institute-European Organisation for Research and Treatment of Cancer; November 14-18, 2005; Philadelphia, PA.

14. Baselga J, Cortés J, Kim SB, et al; CLEOPATRA Study Group. Pertuzumab plus trastuzumab plus docetaxel for metastatic breast cancer. N Engl J Med. 2012;366(2):109-119.

15. Cortés J, Swain SM, Kudaba I, et al. Absence of pharmacokinetic drug-drug interaction of pertuzumab with trastuzumab and docetaxel. Anticancer Drugs. 2013;24(10):1084-1092.

16. Pertuzumab product information. 2012. Available from: http://www. gene.com/download/pdf/perjeta_prescribing.pdf.

17. Baselga J, Gelmon KA, Verma S, et al. Phase II trial of pertuzumab and trastuzumab in patients with human epidermal growth factor receptor 2-positive metastatic breast cancer that progressed during prior trastuzumab therapy. J Clin Oncol. 2010;28(7):1138-1144.

18. Cortés J, Fumoleau P, Bianchi GV, et al. Pertuzumab monotherapy after trastuzumab-based treatment and subsequent reintroduction of trastuzumab: activity and tolerability in patients with advanced human epidermal growth factor receptor 2-positive breast cancer. J Clin Oncol. 2012;30(14):1594-1600.

19. Portera CC, Walshe JM, Rosing DR, et al. Cardiac toxicity and efficacy of trastuzumab combined with pertuzumab in patients with human epidermal growth factor receptor 2-positive metastatic breast cancer. Clin Cancer Res. 2008;14(9):2710-2716.

20. Baselga J, Cortés J, Im SA, et al. Biomarker analyses in CLEOPATRA: a phase III, placebo-controlled study of pertuzumab in human epidermal growth factor receptor 2-positive, first-line metastatic breast cancer. J Clin Oncol. 2014;32(33):3753-3761.

21. Swain SM, Kim SB, Cortés J, et al. Pertuzumab, trastuzumab, and docetaxel for HER2-positive metastatic breast cancer (CLEOPATRA study): overall survival results from a randomised, double-blind, placebo-controlled, phase 3 study. Lancet Oncol. 2013;14(6): $461-471$. 
22. Swain S, Kim S, Cortes J, et al. Final overall survival analysis from the CLEOPATRA study of first-line pertuzumab, trastuzumab, and docetaxel in patients with HER2-positive metastatic breast cancer. Presented at: ESMO 2014; September 28, 2014; Madrid.

23. Swain SM, Baselga J, Miles D, et al. Incidence of central nervous system metastases in patients with HER2-positive metastatic breast cancer treated with pertuzumab, trastuzumab, and docetaxel: results from the randomized phase III study CLEOPATRA. Ann Oncol. 2014;25(6): 1116-1121.

24. Dang C, Iyengar N, Datko F, et al. Phase II study of paclitaxel given once per week along with trastuzumab and pertuzumab in patients with human epidermal growth factor receptor 2-positive metastatic breast cancer. J Clin Oncol. 2015;33(5):442-447.

25. Perez E, Lopez-Vega J, Mastro L, et al. A combination of pertuzumab, trastuzumab, and vinorelbine for first-line treatment of patients with HER2-positive metastatic breast cancer: an open-label, two-cohort, phase II study (VELVET). J Clin Oncol. 2012;30(Suppl):15.

26. Bachelot T, Ciruelos E, Peretz-Yablonski T, et al. First-line pertuzumab, trastuzumab, and taxane therapy for HER2-positive locally recurrent/ metastatic breast cancer: Interim safety results $(\mathrm{N}=704)$ from PERUSE. Presented at: The 50th American Society of Clinical Oncology Annual Meeting; May 30-June 3, 2014; Chicago, IL. Poster \#548.

27. Miller KD, Diéras V, Harbeck N, et al. Phase II trial of trastuzumab emtansine with pertuzumab for patients with human epidermal growth factor receptor 2-positive, locally advanced, or metastatic breast cancer. J Clin Oncol. 2014;32(14):1437-1444.

28. Gianni L, Pienkowski T, Im YH, et al. Efficacy and safety of neoadjuvant pertuzumab and trastuzumab in women with locally advanced, inflammatory, or early HER2-positive breast cancer (NeoSphere): a randomised multicentre, open-label, phase 2 trial. Lancet Oncol. 2012;13(1):25-32.
29. Schneeweiss A, Chia S, Hickish T, et al. Pertuzumab plus trastuzumab in combination with standard neoadjuvant anthracycline-containing and anthracycline-free chemotherapy regimens in patients with HER2positive early breast cancer: a randomized phase II cardiac safety study (TRYPHAENA). Ann Oncol. 2013;24(9):2278-2284.

30. Schneeweiss A, Chia S, Hegg R, et al. Evaluating the predictive value of biomarkers for efficacy outcomes in response to pertuzumab- and trastuzumab-based therapy: an exploratory analysis of the TRYPHAENA study. Breast Cancer Res. 2014;16(4):R73.

31. Swain SM, Ewer MS, Cortés J, et al. Cardiac tolerability of pertuzumab plus trastuzumab plus docetaxel in patients with HER2-positive metastatic breast cancer in CLEOPATRA: a randomized, double-blind, placebo-controlled phase III study. Oncologist. 2013;18(3):257-264.

32. Cortés J, Baselga J, Im YH, et al. Health-related quality-of-life assessment in CLEOPATRA, a phase III study combining pertuzumab with trastuzumab and docetaxel in metastatic breast cancer. Ann Oncol. 2013;24(10):2630-2635.

33. Valachis A, Nearchou A, Polyzos NP. Cardiac toxicity in breast cancer patients treated with dual HER2 blockade. Int J Cancer. 2013;133:2245-2252.

34. Verma S, Miles D, Gianni L, et al; EMILIA Study Group. Trastuzumab emtansine for HER2-positive advanced breast cancer. $N$ Engl J Med. 2012;367:1783-1791.

35. Giordano S, Temin S, Kirshner J, et al; American Society of Clinical Oncology. Systemic therapy for patients with advanced human epidermal growth factor receptor 2-positive breast cancer: American Society of Clinical Oncology Clinical Practice Guideline. J Clin Oncol. 2014;32(19):2100-2108.

36. Cortazar P, Zhang L, Untch M, et al. Pathological complete response and long-term clinical benefit in breast cancer: the CTNeoBC pooled analysis. Lancet. 2014;384(9938):164-172.

\section{Publish your work in this journal}

Breast Cancer: Targets and Therapy is an international, peerreviewed open access journal focusing on breast cancer research, identification of therapeutic targets and the optimal use of preventative and integrated treatment interventions to achieve improved outcomes, enhanced survival and quality of life for the cancer patient.

\section{Dovepress}

View the full aims and scopes of this journal here. The manuscript management system is completely online and includes a very quick and fair peer-review system, which is all easy to use. Visit http:// www.dovepress.com/testimonials.php to read real quotes from published authors. 